\title{
A influência do nível socioeconômico na resolução temporal em escolares
}

\section{The influence of socioeconomic level in temporal resolution in school-age children}

\author{
Sheila Andreoli Balen¹, Mirian Regina Moresco Boeno ${ }^{2}$, Graziela Liebel ${ }^{3}$
}

\begin{abstract}
RESUMO
Objetivo: Verificar a influência do nível socioeconômico na resolução temporal de escolares em dois protocolos de avaliação. Métodos: A amostra foi constituída por 44 crianças de seis a 11 anos, sem histórico de alterações otológicas e/ou audiológicas, de doenças neurológicas e psicológicas conhecidas e com audição normal. A amostra foi dividida em três grupos, de acordo com o Critério de Classificação Econômica do Brasil da Associação Brasileira de Empresas de Pesquisa: Grupo 1: nível socioeconômico alto; Grupo 2: nível socioeconômico médio; Grupo 3: nível socioeconômico baixo. Foram aplicados os testes de detecção de intervalos de silêncio (RGDT) e de detecção de intervalo no ruído (GIN). A análise estatística utilizou o teste Ryan-Einot-Gabriel-Welch Multiple Range Test. Resultados: As médias de desempenho dos grupos 1, 2 e 3 foram maiores no teste de detecção de intervalos de silêncio do que em relação ao de intervalos no ruído. Em relação ao nível socioeconômico, em ambos os testes houve diferenças estatisticamente significativas entre os grupos. Conclusão: Houve influência do nível socioeconômico na resolução temporal medida tanto pelo teste de detecção de intervalos de silêncio quanto pelo de intervalos no ruído.
\end{abstract}

Descritores: Audição; Criança; Testes auditivos; Fatores socioeconômicos; Percepção auditiva

\section{INTRODUÇÃO}

A audição faz parte de um sistema especializado de comunicação, envolvendo muito mais do que apenas a sensibilidade periférica. Este intrincado sentido envolve a participação de redes neuronais complexas e de funções mentais superiores na interpretação de sons verbais e não verbais. Para que tal processo ocorra de forma adequada, é fundamental a integridade do sistema auditivo periférico e central, bem como a exposição à estimulação acústica ${ }^{(1)}$. O processamento auditivo central é definido como os mecanismos e processos envolvidos na localização e lateralização do som, discriminação auditiva, reconhecimento de padrões auditivos, aspectos temporais da audição, ordem e sequência temporal, resolução temporal e desempenho auditivo com sinais acústicos competitivos, em

Trabalho realizado no Curso de Fonoaudiologia da Universidade do Vale do Itajaí - UNIVALI - Itajaí (SC), Brasil, pelo Programa de Iniciação Científica do Artigo $170^{\circ}$ do Governo do Estado de Santa Catarina.

(1) Doutora, Professora do Curso de Fonoaudiologia da Universidade do Vale do Itajaí - UNIVALI - Itajaí (SC), Brasil.

(2) Fonoaudióloga da Prefeitura Municipal de Blumenau - Blumenau (SC), Brasil.

(3) Fonoaudióloga da Prefeitura Municipal de Itapema - Itapema (SC), Brasil Endereço para correspondência: Sheila Andreoli Balen. R. Dr. Abel Capela, 481/103, Coqueiros, Florianópolis (SC), Brasil, CEP: 88080-250. E-mail: sheila@sheilabalen.com.br

Recebido em: 16/12/2008; Aceito em: 27/7/2009 escuta dicótica e de estímulos degradados. O processamento auditivo se refere, então, à eficácia e à eficiência com que o sistema nervoso central utiliza a informação auditiva ${ }^{(2)}$.

Resultados de pesquisas indicam a necessidade de incluir na avaliação e/ou triagem do processamento auditivo os testes que avaliam os aspectos temporais ${ }^{(2)}$. Dentre esses aspectos se destaca a resolução temporal, que consiste no intervalo de tempo mínimo no qual eventos acústicos diferentes podem ser distinguidos. Esses intervalos auxiliam o indivíduo a identificar pequenas variações acústicas que ocorrem no sinal de fala e que lhe permitirão realizar diferentes distinções segmentais, silábicas e de palavras na fala contínua. Alterações na resolução temporal podem resultar em dificuldades para identificar pequenas variações acústicas na fala e, consequentemente, dificuldades em produzir de forma correta os sons da fala ou em interpretar a mensagem ouvida ${ }^{(3)}$.

Entre os procedimentos de avaliação da resolução temporal há o teste de fusão auditiva revisado (Auditory Fusion TestRevised - AFT-R), que mede o limiar de fusão auditiva pela capacidade do ouvinte de identificar a existência de um estímulo ou dois, dependendo da duração do intervalo de silêncio entre os pares apresentados no teste. Outro procedimento é uma modificação do AFT-R denominado teste de detecção de intervalos aleatórios de silêncio (Random Gap Detection Test-RGDT) ${ }^{(4)}$. A modificação introduzida no teste RGDT permitiu apresentar aleatoriamente os intervalos de 
silêncio, que no AFT-R eram apresentados em ordem crescente de duração, isto é, do menor para o maior intervalo de silêncio. Por fim, utiliza-se também o teste de detecção de intervalos no ruído (Gaps-in-noise - GIN), que mede a detecção de intervalos pela interrupção do ruído ${ }^{(5)}$. Nesse teste o ouvinte deve detectar a presença de interrupções de diferentes durações ao longo da apresentação do ruído.

No Brasil, o teste AFT-R foi aplicado em pesquisas com crianças que apresentavam transtornos de déficit de atenção, hiperatividade e de aprendizagem ${ }^{(6)}$; distúrbios de leitura e de escrita ${ }^{(7-9)}$ e fissura palatina ${ }^{(10)}$. O teste RGDT foi utilizado em pesquisas com crianças que apresentavam distúrbios de leitura e escrita ${ }^{(11)}$; desenvolvimento normal ${ }^{(2,12)}$; desvio fonológico ${ }^{(13-14)}$; nascidas a termo e pré-termo ${ }^{(15)}$; com distúrbios do processamento auditivo central e perda auditiva condutiva $^{(16)}$; operadores de telemarketing ${ }^{(17)}$. Já o teste GIN, por ser mais recente, foi estudado apenas em adultos ${ }^{(18-20)} \mathrm{e}$ crianças normais ${ }^{(21)}$.

Observou-se nestas pesquisas que há grande variabilidade na resposta, dependendo do tipo de distúrbio apresentado e do protocolo aplicado, uma vez que a presença de distúrbios de comunicação ou de audição, de forma geral, acarreta alteração na resolução temporal, evidenciada por limiares de detecção de intervalos de silêncio com gaps mais longos. Em sujeitos normais são observadas diferenças entre os desempenhos nos testes RGDT e GIN, sendo que a natureza do estímulo e da tarefa podem justificá-las ${ }^{(18,21)}$.

Em uma pesquisa sobre a influência de paradigmas temporais em testes de processamento temporal auditivo foram encontradas evidências de que variáveis como duração do estímulo e ordem dada pelo avaliador podem interferir no desempenho do indivíduo nesses testes ${ }^{(22)}$. Outra pesquisa de revisão de literatura aponta que os limiares de detecção de gap podem variar de acordo com os seguintes parâmetros: tipos de marcadores, intensidade, frequência e posicionamento do gap nos marcadores, apresentação dos estímulos (mono ou binaural) e efeito de tempo de surgimento e de declínio do sinal ${ }^{(23)}$.

Um estudo sobre a influência do nível socioeconômico e cultural, bem como da estimulação auditiva nas habilidades do processamento auditivo central mostrou que o grupo de crianças de nível socioeconômico baixo apresentou desempenho comparativamente pior, principalmente nos testes de reconhecimento, discriminação e memória sequencial de sons não verbais e verbais ${ }^{(24)}$. Em outra pesquisa relacionada à resolução temporal, 31 crianças de escolas públicas não apresentaram diferenças estatisticamente significantes, comparadas a 43 crianças de escolas particulares. No entanto, foi constatado que a média de limiares de detecção de intervalo de silêncio foi mais longa nas crianças das escolas públicas (média=9,61 ms, DP=3,08) do que das escolas particulares (média=8,08 ms, DP=5,96). Nesta pesquisa também não foram evidenciadas diferenças estatisticamente significantes quanto à variável gênero e idade ${ }^{(12)}$.

As autoras do presente artigo observaram na prática clínica no setor de atendimento Audiológico da Universidade do Vale do Itajaí e durante a realização da pesquisa de campo de outro estudo feito por elas ${ }^{(21)}$ variabilidades individuais entre as crianças submetidas aos testes de resolução temporal (GIN e
RGDT). Essas observações ocorreram a partir da experiência de testar algumas crianças provenientes de escolas municipais, comparando-as a crianças provenientes de escolas particulares. Desta forma, a proposta da pesquisa surgiu da necessidade de estudar a influência do nível socioeconômico na tarefa de resolução temporal para alertar profissionais da área da saúde e da educação no que diz respeito à necessidade de estimulação dessas habilidades no contexto escolar e apontar cuidados importantes nos parâmetros clínicos a serem adotados.

Há carência de estudos na área do processamento auditivo central sobre a variável socioeconômica. No entanto, no contexto do desenvolvimento linguístico, há evidências de que crianças provenientes de lares de classe baixa adquirem a linguagem mais lentamente e mantêm substituições, omissões e distorções de fonemas por mais tempo do que as de classe média ${ }^{(25)}$. O desenvolvimento integral da criança depende tanto dos cuidados que envolvem a dimensão afetiva quanto dos aspectos biológicos do corpo, tais como: qualidade da alimentação, cuidados com a saúde e a forma como ambos são oferecidos à criança ${ }^{(26)}$. Nesse contexto, a estimulação inadequada gerada por influências socioeconômicas e pelo nível educacional da família é um dos fatores que pode contribuir para atrasos no desenvolvimento global da criança, restringindo a aquisição de habilidades motoras, de linguagem e de cognição ${ }^{(27)}$.

Portanto, o objetivo desta pesquisa foi verificar a influência do nível socioeconômico na tarefa de resolução temporal em escolares de seis a 11 anos, em dois protocolos de avaliação: o teste de detecção de intervalos aleatórios de silêncio (Random Gap Detection Test-RGDT) e o teste de detecção de intervalos no ruído (Gaps-in-noise Test - GIN).

\section{MÉTODOS}

A população desta pesquisa se constituiu por crianças de ambos os gêneros, de escolas públicas e particulares de Ensino Fundamental do município de Itajaí (SC), na faixa etária de seis a 11 anos (média=8,88 anos).

A amostra de conveniência foi formada por 44 crianças, sendo 16 do gênero feminino e 28 do gênero masculino, cujos responsáveis consentiram na participação na pesquisa. Essa amostra é denominada de conveniência porque os experimentos clínicos são conduzidos com os próprios pacientes que o clínico/pesquisador tem disponiveis ${ }^{(28)}$. As crianças foram selecionadas entre aquelas encaminhadas para o Setor de Atendimento Audiológico da instituição por duas escolas municipais e uma escola particular do município de Itajaí. Cabe ressaltar que estes encaminhamentos faziam parte da pesquisa e de outras pesquisas realizadas no setor envolvendo crianças com e sem queixas no desenvolvimento de linguagem e/ou de aprendizagem.

A pesquisa foi aprovada pelo Comitê de Ética em Pesquisa da Universidade do Vale do Itajaí com o parecer no . 193/07. Os pais ou responsáveis pelas crianças assinaram o Termo de Consentimento Livre e Esclarecido.

Todas as crianças que foram detectadas com alterações audiológicas foram encaminhadas para avaliação completa otorrinolaringológica, audiológica e do processamento auditivo 
no setor responsável da instituição e, quando necessário, também para avaliação e terapia fonoaudiológica.

Os procedimentos de seleção constaram da aplicação de um questionário no qual havia necessidade de informar: identificação, história auditiva, nível de escolaridade do chefe da família, intercorrências auditivas, desenvolvimento neuropsicomotor e desenvolvimento de linguagem, além de avaliação audiológica, que incluiu meatoscopia, triagem audiométrica nas frequências de $500 \mathrm{~Hz}$ a $4000 \mathrm{~Hz}$ e medidas de imitância acústica em ambas as orelhas. A triagem audiométrica e as medidas de imitância acústica foram realizadas no setor de atendimento Audiológico da instituição. A partir desses procedimentos foram incluídos na pesquisa os sujeitos com as seguintes características: idade entre seis e 11 anos; ausência de histórico otológico e/ou audiológico; ausência de doenças neurológicas, psiquiátricas e psicológicas conhecidas; curva timpanométrica tipo A; resposta a $20 \mathrm{~dB}$ nas frequências de 500 a $4000 \mathrm{~Hz}$ em ambas as orelhas na triagem audiométrica; ausência de alterações na produção articulatória dos fonemas do Português observadas pela fala espontânea da criança ao longo de todo o processo de avaliação; língua portuguesa como primeira e única língua.

Para a identificação do nível socioeconômico foi utilizado um questionário proposto pela Associação Brasileira de Empresas de Pesquisa (ABEP) ${ }^{(29)}$, que tem a função de estimar o poder de compra das famílias. O questionário foi entregue aos pais dos sujeitos selecionados para que fosse respondido durante uma das sessões de testes a que as crianças compareceram. A aplicação do questionário teve como objetivo classificar o nível socioeconômico de cada criança com base no nível de instrução do provedor principal da casa e características de moradia de acordo com o Critério de Classificação Econômica do Brasil da $\mathrm{ABEP}^{(29)}$.

O questionário é composto por duas questões gerais, divididas em itens. Na primeira questão foi solicitado aos pais ou responsáveis que respondessem quantos e quais destes itens possuíam em sua residência: televisão, rádio, banheiro, automóvel, empregada/mensalista, aspirador de pó, máquina de lavar, videocassete e/ou DVD, geladeira, freezer (aparelho independente ou parte da geladeira duplex). Na segunda questão se solicitou o grau de instrução do chefe da família, de acordo com a seguinte classificação: analfabeto, primário incompleto, primário completo, ginasial incompleto, ginasial completo, colegial incompleto, colegial completo, superior incompleto e superior completo. Esses termos foram mantidos conforme o documento original da $\mathrm{ABEP}^{(29)}$.

Para a análise do questionário socioeconômico foram utilizados os Critérios de Classificação Econômica Brasil, usando o sistema de pontuação para os itens de cada questão citada anteriormente. As pontuações descritas pela ABEP foram: entre 25 e 34 pontos, classes A1 e A2; entre 17 e 24 pontos, classes $\mathrm{B} 1, \mathrm{~B} 2$ e C; entre 0 e 10 pontos, classes $\mathrm{D}$ e $\mathrm{E}^{(29)}$.

Com o intuito de auxiliar na clareza textual, o presente estudo utilizou os seguintes termos: Grupo 1: nível socioeconômico alto (classes A1 e A2); Grupo 2: nível socioeconômico médio (classes B1, B2 e C) e Grupo 3: nível socioeconômico baixo (classes D e E). Assim, foram classificadas no Grupo 1 oito crianças, 25 crianças no Grupo 2, e 11 no Grupo 3.
O Grupo 1 se formou com quatro meninas e quatro meninos, com média de idade de 8,87 anos ( $\mathrm{DP}=1,45)$. O Grupo 2 foi constituído por 16 meninos e nove meninas com média de idade de 8,84 anos ( $\mathrm{DP}=1,62)$, e o Grupo 3 por oito meninos e três meninas com média de idade de 9,0 anos $(\mathrm{DP}=1,41)$.

Os procedimentos de pesquisa aplicados foram o teste de detecção de intervalos aleatórios de silêncio (Random Gap Detection Test - RGDT) e o teste de detecção de intervalos no ruído (Gaps-in-noise Test-GIN). Ambos foram realizados utilizando um Compact Disc Player acoplado a um audiômetro Interacoustic ${ }^{\circledR} \mathrm{AC}-33$ ou AC-40.

$\mathrm{O}$ teste RGDT consiste em pares de tons puros nas frequências de 500, 1000, 2000 e $4000 \mathrm{~Hz}$, com intervalos entre os dois tons que variam de 0 a $40 \mathrm{~ms}$ (RGDT) e de 50 a 300 ms (RGDT-expandido). Cada criança foi instruída a responder gestualmente se ouvia um ou dois tons. O teste foi apresentado a $50 \mathrm{~dB}$ NS nas frequências testadas na condição binaural. Inicialmente, realizou-se o treino de 0 a $40 \mathrm{~ms}$ com apresentação crescente dos intervalos, na faixa de frequência de $500 \mathrm{~Hz}$. Caso a criança detectasse intervalos nessa faixa de duração, dava-se início à pesquisa do limiar de detecção de intervalos no silêncio de 0 a $40 \mathrm{~ms}$, apresentados aleatoriamente, nas frequências de 500, 1000, 2000 e $4000 \mathrm{~Hz}$. Caso a criança não identificasse nenhum dos intervalos como dois tons durante o treino, dava-se continuidade ao teste utilizando o RGDT-Expandido, medindo as mesmas frequências, porém com intervalos de até $300 \mathrm{~ms}$. O limiar de detecção do gap foi caracterizado pelo menor intervalo a partir do qual o indivíduo passou a identificar a presença de dois estímulos. Foram calculados o limiar de cada frequência e a média dos limiares das quatro frequências. O tempo médio de aplicação do teste foi de 10 minutos.

$\mathrm{Na}$ análise do RGDT calculou-se a média e o desvio padrão do limiar de detecção de gap da amostra em 500, 1000, 2000 e $4000 \mathrm{~Hz}$, bem como o valor de média do limiar de detecção de gap de cada sujeito da amostra.

O teste GIN é composto por vários segmentos de ruído branco de seis segundos que contem de zero a três intervalos de silêncio cada. Cada segmento de ruído é separado de outro por cinco segundos de silêncio (intervalo entre os estímulos) e as durações dos gaps são 2, 3, 4, 5, 6, 8, 10, 12, 15 e 20 ms. Cada uma dessas durações aparece seis vezes ao longo de cada faixa de teste. Tanto a duração quanto a localização dos gaps dentro dos segmentos de ruído são pseudorandomizados em relação à sua ocorrência. Existem dez itens para treino, que precedem o início dos itens para o teste ${ }^{(20)}$. Neste estudo foi utilizada, inicialmente, a faixa de treino. A faixa de teste seguinte foi utilizada apresentando os estímulos à orelha direita e a subsequente à orelha esquerda. Esta ordem de aplicação foi mantida em toda a amostra. Ressalta-se que o teste GIN é composto de quatro faixas de testes, além do treino. No entanto, já foi evidenciada que não há diferença entre as orelhas investigadas e as faixas apresentadas. Desta forma, é suficiente para a investigação do limiar de detecção do gap o uso de uma faixa de teste para cada orelha ${ }^{(19-20)}$.

As crianças foram orientadas a apertar um botão quando o ruído contínuo monoaural que ouviam fosse interrompido por um intervalo de silêncio. 
Como critério para determinar o limiar de detecção de gap no teste GIN foi observada qual a menor duração do gap no qual a criança detectou quatro das seis apresentações contidas em cada faixa de teste por orelha ${ }^{(5)}$.

Realizou-se análise estatística descritiva para caracterizar a amostra por frequência testada no RGDT e de acordo com o limiar de detecção do gap em cada orelha no GIN, comparando essas variáveis entre os grupos de faixa socioeconômica (Grupo 1, Grupo 2 e Grupo 3).

Para o teste RGDT foram postas à prova as hipóteses referentes às variáveis nível socioeconômico e frequências em Hz. Também foi realizada a análise não paramétrica com o teste de Wilcoxon para frequências dentro de cada grupo e de grupos dentro de cada frequência.

Para o teste GIN foram testadas as hipóteses referentes às variáveis nível socioeconômico e orelha. O teste de Wilcoxon também foi aplicado para verificar se havia diferença entre as orelhas.

Estas hipóteses foram submetidas à análise de variância, utilizando o teste de separação de médias Ryan-Einot-GabrielWelch Multiple Range Test, com nível de probabilidade de erro $\mathrm{p}=0,05$. Tudo dentro de um modelo matemático adequado para cada teste, visando o controle do erro experimental.

\section{RESULTADOS}

A menor média para o teste de detecção de intervalos aleatórios de silêncio (RGDT) ocorreu no Grupo 1 para todas as frequências, sendo que o Grupo 3 apresentou maiores médias nos limiares de detecção dos intervalos em todas as frequências (Tabela 1).

Tabela 1. Teste de detecção de intervalos aleatórios de silêncio (RGDT) em ms por grupos de frequências

\begin{tabular}{lccccc}
\hline Nível & \multicolumn{5}{c}{ Frequências em Hz } \\
\cline { 2 - 6 } socioeconômico & 500 & 1000 & 2000 & 4000 & Médias \\
\hline Grupo 1 & 24,00 & 29,00 & 24,63 & 38,13 & 28,94 \\
Grupo 2 & 41,50 & 54,77 & 61,92 & 43,85 & 50,51 \\
Grupo 3 & 75,00 & 98,00 & 78,50 & 82,00 & 83,38 \\
\hline Médias & 45,95 & 59,91 & 58,91 & 51,48 & 54,06
\end{tabular}

Legenda: RGDT = random gap detection test

Constatou-se que ocorreram diferenças estatisticamente significantes entre os grupos 1,2 e 3 ( $p=0,0005$ ).

No entanto, ao analisar a influência da frequência na média dos limiares de detecção dos intervalos nos três grupos, não se observa evidência de diferenças estatisticamente significantes.

O Grupo 1 apresentou melhor desempenho no teste de detecção de intervalo no ruído (GIN) para ambas as orelhas (OE e OD), em relação aos grupos 2 e 3 . Além disso, também se observa melhor desempenho do Grupo 2 ao compará-lo com o Grupo 3. Essa diferença foi estatisticamente significante entre todos os grupos ( $\mathrm{p}=0,0001)$ (Tabela 2).

Em relação à variável orelha (lista $1 \mathrm{x}$ lista 2) não houve diferença estatisticamente significante.

Quanto à relação da média total dos grupos 1, 2 e 3 para as frequências testadas no RGDT e a média das orelhas direita
Tabela 2. Teste de detecção de intervalos no ruído (GIN) em ms por grupos e orelhas avaliadas

\begin{tabular}{lccc}
\hline Nível socioeconômico & $\begin{array}{c}\text { Orelha } \\
\text { direita }\end{array}$ & $\begin{array}{c}\text { Orelha } \\
\text { esquerda }\end{array}$ & Médias \\
\hline Grupo 1 & 6,38 & 5,63 & 6,00 \\
Grupo 2 & 7,68 & 7,08 & 7,38 \\
Grupo 3 & 9,10 & 9,30 & 9,20 \\
\hline Médias & 7,77 & 7,33 & 7,55 \\
\hline
\end{tabular}

Legenda: $\mathrm{GIN}=$ gaps in noise test

e esquerda para os grupos 1, 2 e 3 no GIN, os resultados evidenciaram que a média geral do limiar de detecção de intervalos de silêncio (RGDT) foi maior em relação à média de detecção de intervalos no ruído (GIN). Salienta-se que a amostra estudada foi composta pelos mesmos sujeitos para a realização de ambos os testes (Figura 1). Não foram evidenciadas diferenças estatisticamente significantes $(p>0,05)$ nos resultados por frequência no teste RGDT e por orelha no teste GIN, utilizando o teste Wilcoxon.

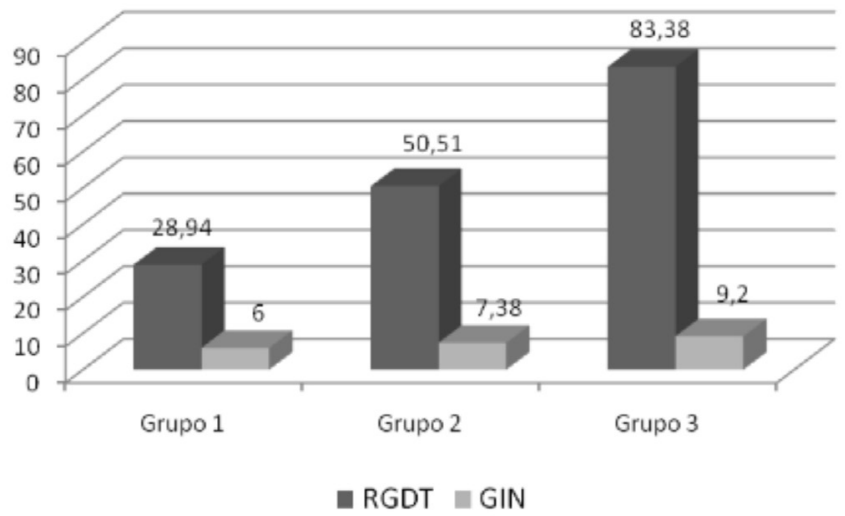

Legenda: $\mathrm{RGDT}$ = random gap detection test; $\mathrm{GIN}=$ gaps in noise test

Figura 1. Desempenho médio dos limiares do teste de detecção de intervalos no silêncio (RGDT) e de ruído (GIN) por grupo

Os resultados dos testes RGDT e GIN em relação ao nível socioeconômico conduziram à mesma conclusão, isto é, ambos demonstraram diferenças estatisticamente significantes entre os três grupos. Salienta-se, porém, que o coeficiente de variação (CV) no teste GIN foi de 29,96564 e no RGDT de 61,10140. Desta forma, no teste GIN se constata menor variabilidade do que no teste RGDT (Figura 1).

\section{DISCUSSÃO}

Os limiares de duração dos intervalos de silêncio (RGDT) por frequência, encontrados na amostra estudada (Tabela 1), foram semelhantes aos resultados de estudos com crianças com desvio fonológico ${ }^{(13-14)}$, distúrbio de processamento auditivo e perda auditiva condutiva ${ }^{(16)}$, bem como aos estudos de crianças com distúrbio de leitura ${ }^{(11)}$.

A semelhança dos três grupos de níveis socioeconômicos no teste RGDT se deu de forma diferenciada em relação aos estudos citados anteriormente ${ }^{(11,13-14,16)}$. O Grupo 1, de nível socioeconômico alto, apresentou compatibilidade com os 
resultados encontrados nos estudos com crianças com distúrbio de leitura e escrita e crianças com desvio fonológico ${ }^{(11,14)}$. O Grupo 2, de nível socioeconômico médio, teve desempenho semelhante ao encontrado em estudos com crianças que apresentam distúrbio de processamento auditivo central ${ }^{(16)} \mathrm{e}$ com crianças que apresentam desvio fonológico ${ }^{(13)}$. Já o Grupo 3 teve desempenho semelhante a estudo com crianças que apresentam perda auditiva condutiva ${ }^{(16)}$.

Ao comparar os resultados do teste RGDT encontrados nesta pesquisa aos de estudos com crianças brasileiras em desenvolvimento normal ${ }^{(1,11-12,14,16,21)}$, observa-se que os limiares de detecção de intervalos de silêncio (RGDT) obtidos neste estudo foram maiores para todos os níveis socioeconômicos considerados. Ressalta-se que, no presente estudo, os sujeitos não foram separados por parâmetros de normalidade ou presença de alterações no processamento auditivo, tendo em vista que não fazia parte do objetivo das autoras. Assim, a diferença encontrada entre esta e as pesquisas relatadas pode ter ocorrido porque essa variável não foi controlada, havendo necessidade de investigar a relação entre o nível socioeconômico e a presença/ausência de distúrbios no processamento auditivo central em estudos futuros.

Os estudos utilizando o teste RGDT com crianças escolares americanas normais ${ }^{(4,30)}$ demonstraram parâmetros de normalidade de $7,07 \mathrm{~ms}^{(4)}$ e de $4,77 \mathrm{~ms}^{(30)}$, o que indica disparidade em relação a todos os grupos desta pesquisa e em relação a outras pesquisas realizadas no Brasil também com crianças normais $^{(1,11-12,14,16,21)}$. Esta disparidade pode ter ocorrido pela influência de fatores relacionados à língua materna, ao nível socioeconômico, nutricionais, de desenvolvimento do sistema nervoso auditivo central e ao nível de escolaridade das crianças, bem como pode ser resultado de diferentes critérios de normalidade utilizados entre as pesquisas para seleção e constituição das amostras de crianças normais.

No teste GIN, os resultados do Grupo 1 evidenciaram que os limiares de detecção de gap encontrados em cada orelha foram semelhantes aos encontrados em adultos normais ${ }^{(5,18-20)}$. Um estudo que utilizou o teste GIN em crianças normais em idade escolar ${ }^{(21)}$ obteve limiar de detecção médio entre as orelhas de 5,5 ms. Esse resultado é semelhante ao obtido pelo Grupo 1 (6 ms) da pesquisa e difere dos resultados dos Grupos 2 e 3, que apresentaram limiares maiores. Ressalta-se que esta investigação não evidenciou diferenças no desempenho entre as orelhas, o que corrobora outros estudos ${ }^{(7,18-21)}$.

É possível que o nível socioeconômico interfira no desenvolvimento da resolução temporal, tendo em vista a semelhança entre a média do limiar de detecção de gap do Grupo 1 e o das crianças ${ }^{(21)}$ e adultos normais ${ }^{(5,18-20)}$. No entanto, os achados desta pesquisa não permitem concluir que o nível socioeconômico baixo possa gerar alterações na resolução temporal. São necessárias novas investigações com ampliação da amostra e controle de outras variáveis como a presença e ausência de distúrbios do processamento auditivo central.

Quanto às relações entre os testes, no teste GIN é possível observar também a diferença de desempenho das crianças nos três grupos de níveis socioeconômicos. Porém, o limiar de detecção de intervalo de silêncio no ruído (GIN) foi menor se comparado com o limiar encontrado no teste de detecção de intervalo aleatório de silêncio (RGDT). Um fator que pode justificar a grande variabilidade de resposta dos sujeitos no teste RGDT é o critério utilizado para estabelecer o limiar de detecção de gap. No RGDT são apresentados apenas uma vez intervalos entre os tons com durações que variam de 0 a $40 \mathrm{~ms}$ ou de 50 a $300 \mathrm{~ms}$ (RGDT-expandido). O limiar é determinado pela menor duração na qual o ouvinte detectou dois tons, evidenciando que identificou o intervalo de duração entre eles. Desta forma, há apenas uma chance para cada duração apresentada.

Já no teste GIN são apresentados por seis vezes os intervalos, que variam de 2 a $20 \mathrm{~ms}$, sendo estabelecido o limiar de detecção de gap como aquele no qual o sujeito identificou quatro das seis apresentações. Assim sendo, é estabelecido um percentual de detecção do gap, buscando qual é o intervalo detectado de no mínimo $66,67 \%$ no teste GIN. Por outro lado, no teste RGDT já é exposto como $100 \%$ ou $0 \%$ em cada intervalo de duração entre os tons, havendo maior rigidez no critério do referido teste. Acredita-se, portanto, que as diferenças entre os resultados obtidos nos dois testes possam ser decorrentes da especificidade dos testes, assim como apontam outros autores ${ }^{(5,19)}$.

Estes achados estão de acordo com os observados em outros estudos ${ }^{(18,21,30)}$, que evidenciaram a interferência das diferenças entre os tipos de estímulos utilizados em cada teste (ruído branco no teste GIN e tom puro no teste RGDT) e o tipo de resposta requerida nos testes (apertar um botão ou apenas indicar cada vez que um gap é percebido no teste GIN e informar quantos sons foram detectados no RGDT). A questão do tipo de resposta utilizada em cada teste também maximiza possíveis confusões durante sua execução, já que, como ocorre no RGDT, contar o número de estímulos ou responder verbalmente pode requerer maiores habilidades cognitivas. Salienta-se que o GIN avalia separadamente os canais auditivos direito e esquerdo, o que é uma informação importante na avaliação de crianças e idosos, uma vez que pode haver diferença entre as orelhas em sujeitos com distúrbios das vias auditivas centrais ${ }^{(5)}$. Por esse motivo, o protocolo do GIN foi proposto com aplicação diferenciada para as duas orelhas.

Em estudo desenvolvido com crianças de escola particular e pública ${ }^{(12)}$ evidenciou-se que não houve diferença estatisticamente significante entre os desempenhos dos dois grupos de sujeitos no teste RGDT, porém, as crianças de rede pública apresentaram limiares de detecção de intervalo de silêncio discretamente maiores do que os apresentados pelas crianças da escola particular. O presente estudo corrobora em parte esses resultados, mesmo não sendo o nível socioeconômico o principal objetivo da pesquisa citada $^{(12)}$, já que foi uma das poucas pesquisas a relacionar as possíveis questões socioeconômicas dos grupos da pesquisa, classificando-as por escola pública e privada. É possível que a diferença entre os resultados das pesquisas se deva à diferença entre os parâmetros utilizados para classificar o nível socioeconômico, uma vez que atualmente é comum encontrar crianças de níveis socioeconômicos médios em escolas públicas, bem como, pela concessão de bolsas de estudo, encontrar crianças de níveis socioeconômicos baixos em escolas particulares. Desta forma, a separação por tipo de 
escolas nem sempre é o indicador mais real para identificar e classificar o nível socioeconômico nos dias atuais.

\section{CONCLUSÃO}

Com base nos resultados desta pesquisa pode-se concluir que o nível socioeconômico pode influenciar a resolução temporal, medida tanto pelo teste RGDT quanto pelo teste GIN. Porém, são necessárias novas pesquisas, controlando outras variáveis, para verificar a correlação entre a presença de distúrbios de processamento auditivo central ou outros transtornos de linguagem e aprendizagem e o nível socioeconômico. Da mesma maneira, estudos normativos com amostras maiores em cada faixa-etária também devem incluir a variável nível socioeconômico, no intuito de estabelecer os critérios de normalidade para a população infantil brasileira, estudando suas possíveis influências.

Os resultados apresentados neste estudo sugerem a necessidade de estímulo das habilidades de resolução temporal, o que pode ser realizado no próprio contexto escolar.

\begin{abstract}
Purpose: To verify the influence of socioeconomic status in temporal resolution of school-age children in two evaluation protocols. Methods: The sample comprised 44 children with ages ranging from six to 11 years old; all of them had no history of otological and/ or audiological alterations, known neurological and psychological diseases, and had normal hearing. The sample was divided into three groups, according to the Brazilian Criterion of Economic Classification of the Brazilian Research Enterprises Association: Group 1: high socioeconomic level; Group 2: middle socioeconomic level; Group 3: low socioeconomic level. The random gap detection test (RGDT) and the gaps-in-noise test (GIN) were carried out. Statistical analysis was conducted using the Ryan-Einot-GabrielWelch Multiple Range Test. Results: Mean scores of groups 1, 2 and 3 were higher for the RGDT than for the GIN test. Regarding socioeconomic status, both tests showed statistically significant differences among the groups. Conclusion: Socioeconomic status influenced temporal resolution measured by both RGDT and GIN tests.
\end{abstract}

Keywords: Hearing; Child; Hearing tests; Socioeconomic factors; Auditory perception

\title{
REFERÊNCIAS
}

1. Barreto MA, Muniz LF, Teixeira CF. Desempenho da habilidade a resolução temporal em crianças de 07 a 13 anos. Rev Soc Bras Fonoaudiol. 2004;9(4):220-8.

2. American Speech-Language-Hearing Association. (Central) Auditory processing disorders. Technical report [internet] 2005. [cited 2006 Mar 25] Available from: http://www.asha.org/docs/html/TR2005-00043.html

3. Balen SA. Processamento auditivo central: aspectos temporais da audição e percepção acústica da fala 1997 [mestrado]. São Paulo: Pontifícia Universidade Católica de São Paulo; 1997.

4. Keith RW. Random gap detection test. St Louis: Auditec; 2000.

5. Musiek FE, Shinn JB, Jirsa R, Bamiou DE, Baran JA, Zaida E. GIN (Gaps-In-Noise) test performance in subjects with confirmed central auditory nervous system involvement. Ear Hear. 2005;26(6):608-18.

6. Feniman MR, Keith RW, Cunningham RF. Assessment of auditory processing in children with attention deficit hyperactivity disorder and language-based learning impairments. Distúrb Comun. 1999;11(1):9-27.

7. Moura DR, Feniman MR, Lauris JR. Teste de fusão auditiva - revisado em crianças com distúrbios de leitura e escrita. J Bras Fonoaudiol. 2000;3:38-43

8. Garcia VL. Processamento auditivo em crianças com e sem distúrbios de aprendizagem [doutorado]. São Paulo: Universidade Federal de São Paulo; 2001.

9. Costa LP, Pereira LD, Santos MF. Auditory fusion test in scholars. PróFono. 2004;16(2):187-96.

10. Cassab TV, Zorzetto NL. Teste da fusão auditiva-revisado (AFT-R) em crianças com fissura labiopalatina. Acta AWHO. 2002;21(3/4):25-32.

11. Branco-Barreiro FC. Estudo do processamento auditivo temporal em alunos de escola pública com e sem dificuldade de leitura [doutorado]. São Paulo: Universidade de São Paulo; 2003.

12. Dias AM. Desempenho de escolares para o teste de detecção de intervalo de silêncio em tom puro [mestrado]. São Paulo: Pontifícia Universidade Católica de São Paulo; 2004.
13. Ventriglio PR. Estudo do processamento auditivo temporal de crianças com desvio fonológico [mestrado]. São Paulo: Pontifícia Universidade Católica de São Paulo; 2005.

14. Muniz LF, Roazzi A, Schochat E, Teixeira CF, de Lucena JA. Avaliação da habilidade de resolução temporal, com uso do tom puro, em crianças com e sem desvio fonológico. Rev CEFAC. 2007;9(4):550-62.

15. Fortes AB, Pereira LD, Azevedo MF. Resolução temporal: análise em pré-escolares nascidos a termo e pré-termo. Pró-Fono. 2007;19(1):87-96.

16. Balen SA, Bretzke LM, Mottecy CM, Liebel G, Boeno MR, Gondim LM. Resolução temporal de crianças: comparação entre audição normal, perda auditiva condutiva e distúrbio do processamento auditivo. Rev Bras Otorrinolaringol. 2009;75(1):123-9.

17. da Silva MC, Cunha MB, Souza CC, Mitre EI. Avaliação do processamento auditivo em operadores de telemarketing. Rev CEFAC. 2006;8(4):536-42.

18. Zaidan E, Garcia AP, Tedesco ML, Baran JA. Desempenho de adultos jovens normais em dois testes de resolução temporal. Pró-Fono. 2008;20(1):19-24.

19. Samelli AG, Schochat E. The gaps-in-noise test: gap detection thresholds in normal-hearing young adults. Int J Audiol. 2008;47(5):238-45.

20. Samelli AG, Schochat E. Estudo da vantagem da orelha em teste de detecção de gap. Rev Bras Otorrinolaringol. 2008;74(2):235-40.

21. Balen SA, Liebel G, Boeno, MR, Mottecy CM. Resolução temporal de crianças escolares. Rev CEFAC. 2009;11(supl. 1):52-61.

22. Murphy CF, Schochat E. Influência de paradigmas temporais em testes de processamento temporal auditivo. Pró-Fono. 2007;19(3):259-66.

23. Samelli AG, Schochat E. Processamento auditivo, resolução temporal e teste de detecção de gap: revisão da literatura. Rev CEFAC. 2008;10(3): 369-77.

24. Almeida CC, Lopes CC, Machado LM, Gadel M, Costa M, Pereira LD. Influência do nível sócio econômico e cultural e da estimulação auditiva nas habilidades do processamento auditivo central. Fono Atual. 1997;1(2):12-17. 
25. Cazden CB. Three sociolinguistic views of the language and speech of lower-class children with special attention to the work of Basil Bernstein. Dev Med Child Neurol. 1968;10(5):600-12.

26. Lima MC, Barbarini GC, Gagliardo HG, Arnais MA, Gonçalves VM. Observação do desenvolvimento de linguagem e funções auditiva e visual em lactentes. Rev Saúde Pública. 2004;38(1):106-12.

27. Azevedo MF, Vieira RM, Vilanova LC. Desenvolvimento auditivo de crianças normais e de alto risco. São Paulo: Plexus; 2001.
28. Vieira S, Hossne WS. Metodologia científica para a área da saúde. Rio de Janeiro: Campos; 2001.

29. Associação Brasileira de Empresas de Pesquisa - ABEP (2003). Critérios de Classificação Econômica Brasil [Internet] 2003 [citado 2007 Abr 19]. Disponível em: http://www.abep.org/novo/Content.aspx?ContentID=302

30. Chermak GD, Lee J. Comparison of children's performance on four tests of temporal resolution. J Am Acad Audiol. 2005;16(8):554-63. 